\title{
The First Occurrence of Certain Large Prime Gaps
}

\author{
By Richard P. Brent
}

\begin{abstract}
The first occurrence of a string of $2 r-1$ consecutive composite numbers between two primes (denoted by $f(r)$ and $f(r)+2 r$ ) is tabulated for $f(r)$ in the range $2.6 \times 10^{12}<f(r) \leqslant 4.444 \times 10^{12}$. This extends earlier computations in the range $f(r) \leqslant 2.6 \times 10^{12}$.
\end{abstract}

Let $p_{1}=2, p_{2}=3, \ldots$ be the sequence of primes. For integer $r \geqslant 1$, define

$$
f(r)= \begin{cases}p_{j} & \text { if } j \geqslant 1 \text { is minimal such that } p_{j+1}-p_{j}=2 r \\ \infty & \text { if no such } j \text { exists. }\end{cases}
$$

See [1] and [3] for a discussion of the asymptotic behavior of $f(r)$, and for additional references. All $f(r) \leqslant 2.6 \times 10^{12}$ are tabulated in [1] and [2]. In Table 1 we give all eleven values of $f(r)$ in the range $2.6 \times 10^{12}<f(r) \leqslant 4.444 \times 10^{12}$.

The maximal gap has $r=326$, i.e. $p_{j+1}-p_{j} \leqslant 652$ for all $p_{j} \leqslant 4.444 \times 10^{12}$, and $p_{j+1}-p_{j}=652$ for $p_{j}=2,614,941,710,599$. The minimal $r$ for which $f(r)$ is still unknown is $r=268$, and the next is $r=279$.

The computation of Table 1 was performed over the period April 1973 to September 1978, on an IBM 360/50 computer with $256 \mathrm{~K}$ bytes of memory. The method used was the same as that described in [1], except that the sieve size had to be reduced to fit into the $208 \mathrm{~K}$ bytes available. About 61 seconds were required to sieve each block of $2,661,120$ numbers near $3 \times 10^{12}$.

TABLE 1

\begin{tabular}{cccc}
\hline$r$ & $f(r)$ & $r$ & $f(r)$ \\
\hline 271 & 2707053887651 & 285 & 4442109925217 \\
272 & 2652427555639 & 287 & 3108794067079 \\
274 & 3380058341279 & 296 & 3410069454097 \\
277 & 3621153039299 & 309 & 4165633395149 \\
278 & 4338624362173 & $326^{*}$ & 2614941710599 \\
280 & 4260199366373 & &
\end{tabular}

Received March 28, 1980.

1980 Mathematics Subject Classification. Primary 10-04, 10A20, 10A25, 65A05.

Key words and phrases. Distribution of primes, prime gap, maximal prime gap, successive composites, consecutive primes. 
Department of Computer Science

Australian National University

Canberra, A.C.T. 2600, Australia

1. R. P. BRENT, "The first occurrence of large gaps between successive primes," Math. Comp., v. 27, 1973, pp. 959-963. MR 48 \#360.

2. L. J. LANDER \& T. R. PARKIN, "On the first appearance of prime differences," Math. Comp., v. 21, 1967, pp. 483-488. MR 37 \#6237.

3. D. SHANKS, "On maximal gaps between successive primes," Math. Comp., v. 18, 1964, pp. 646-651. MR 29 \#745. 